\title{
TUMOR DE KRUKENBERG DEL OVARIO
}

\section{A propósito de una historia del Hospital San Juan de Dios. - Cúcuta.}

\author{
Dr. Alberto Duarte-Contreras, M.D.* \\ Dr. Luis Fernando Luzardo M., M.D.*
}

El tumor de Krukenberg ha despertado poco interés entre nosotros, tal vez por ser una entidad relativamente rara; sólamente se conoce un trabajo en la bibliografía nacional (14). Esto nos ha motivado a publicar algunas observaciones en torno a una historia clínica de nuestro servicio.

\footnotetext{
"Historia Clínica 040820. Mujer de 40 años; menarca a los 16; ciclos normales 28/3. G2 P2. Dos meses después del segundo parto ingresa al Departamento Quirúrgico de nuestro Hospital con sintomatología de obstrucción pilórica. Se practica gastrectomía el 4 de Marzo por "adenocarcinoma gástrico" (M. 2721). Diez días más tarde se da de alta; no asiste a controles. Dos años después consulta por precario estado general, dolor abdominal y presencia de una masa en fosa ilíaca derecha e hipogastrio, de crecimiento relativamente rápido. Se interviene con diagnóstico de "tumor maligno de ovario, posible tumor de Krukenberg". Se encuentra una masa dependiente de ovario derecho, no adherida, de 7 por 8 por $4 \mathrm{ctm}$. de superficie nacarada con zonas violáceas, multilobular, dura. En ovario izquierdo hay otra masa de caracteres similares, de 7 por $5 \mathrm{cmt}$. Se extraen $150 \mathrm{cc}$. de líquido ascítico. Se practica histerectomía total con salpingo ooforectomía bilateral. La descripción microscópica indica: "ambos ovarios están invadidos por tumoración metastásica; se aprecia estroma fibroso con infiltración difusa de células tumorales y glándulas de contenido mucinoso. Espacios quísticos de contenido mucinoso. Tumor de Krukenberg" (M. 5962). Siete días más tarde se da de alta por exigencia de la paciente, en condiciones poco aceptables. No vuelve a control y un año
}

más tarde fallece en clínica particular con metástasis pulmonar".

\section{Generalidades}

En 1896 Federico Evaristo Krukenberg describió un tumor ovárico que denominó "fibrosarcoma ovarii mucocelullare carcinomatodes". En 1902 Schalangenhaufer descubrió la verdadera naturaleza del tumor, identificó su génesis epitelial y demostró que es un foco secundario de un carcinoma alejado procedente de ordinario del tubo gastrointestinal y lo denominó como "metastatisches ovarial karzinom" (17).

Su origen a nivel de un teratoma, o de la degeneración mucoide de un tumor de Brenner, o de quistes seudomucinosos, bien pudiera indicarnos que estas entidades adecúan el medio ovárico para su desarrollo.

\section{Frecuencia}

Su incidencia es baja; sin embargo entre nosotros lo encontramos entre el 2.7 y el $11.77 \%$ de las neoplasias ováricas.

* Del Departamento Gineco-Obstétrico del Hospital San Juan de Dios. Cúcuta, Colombia. 
TUMOR DE KRUKENBERG DEL OVARIO.

FRECUENCIA EN CINCO HOSPITALES DEL PAIS

\begin{tabular}{|c|c|c|c|c|}
\hline Hospital & Años & $\begin{array}{l}\text { Total de tumores } \\
\text { Malignos de ovario }\end{array}$ & $\begin{array}{l}\text { Total de tumores } \\
\text { de Krukenberg }\end{array}$ & $\%$ \\
\hline $\begin{array}{l}\text { Instituto Nacional de Can- } \\
\text { cerología, Bogotá (7) } \\
\text { Universitario San José. }\end{array}$ & $1957-1970$ & 443 & 12 & 2.7 \\
\hline $\begin{array}{l}\text { Popayán (5) } \\
\text { Universitario de Caldas. }\end{array}$ & $1954-1970$ & 44 & 2 & 4.54 \\
\hline Manizales $(20)$ & $1965-1970$ & 32 & 2 & 4.45 \\
\hline $\begin{array}{l}\text { San Juan de Dios. Cúcuta. } \\
\text { San Juan de Dios. }\end{array}$ & $1960-1971$ & 13 & 1 & 7.69 \\
\hline Bogotá ( 12$)$. & $1957-1970$ & $\begin{array}{l}187 \\
719\end{array}$ & $\begin{array}{l}22 \\
39\end{array}$ & $\begin{array}{r}11.77 \\
6.63\end{array}$ \\
\hline
\end{tabular}

En México, al estudiar 492 neoplasias malignas de ovario encontraron sólo un tumor de Krukenberg $(0.29 \%)$ (26).

Se le puede encontrar concomitante con embarazo (13): Diddle en 1955 recopiló 26 casos (4) y Woodruff informó en 1960 sobre otros doce en gestantes (27). Todos los recién nacidos fueron una franca respuesta al estado general de las pacientes y algunos presentaron alteraciones endocrinas $(2,6)$.

\section{Edad}

Se presenta en pacientes que van descle los 14 años (15) hasta edad muy avanzada. En la casuística colombiana que presentamos la paciente de menor edad tenía 20 años y la mayor era de $57(5,7,12,20)$.

\section{Clasificación}

Fuede ser primario o secundario. Es primario en el $20 \%$ de los casos (27). Se le clasifica como tumor primario si se comprueba la ausencia de toda otra lesión extraovárica por la autopsia o por el seguimiento de la paciente por un tiempo no menor de cinco años (10).
El tumor secundario lo es a un tumor del tubo gastrointestianl 0 a un tumor del seno. Rara vez el tumor primitivo está localizado en vesícula biliar o en sigmoide (1). Linard al revisar la literatura universal recopila 530 tumores de Krukenberg y encuentra que son secundarios a un tumor del estómago en el $70 \%$, a un tumor del intestino grueso en el $15 \%$ $y$ a un tumor del seno en el $6 \%$ (11). Soloway al estudiar una pequeña serie de 16 casos encuentra que 4 ( $25 \%$ ) son secundarios a tumor del seno (23). Parsons dice que este porcentaje se sube hasta el $60 \%$ cuando el tumor del seno se acompaña de metástasis ósea (21).

No todos los tumores ováricos secundarios a un cáncer gastroentérico - del seno son tumores de Krukenberg; para ello es indispensable llenar una serie de requisitos anatomopatológicos que veremos a continuación.

\section{Anatomía patológica}

Macroscópicamente son tumoraciones duras, bilaterales en el 50 a $90 \%$ de los casos (10), sólidas, de forma arriñonada $u$ ovoide. La superficie es lisa o nodular, de aspecto pálido. Al corte se aprecia una coloración amraillenta homogénea, o rojiza, o 
grisácea, compacta, con algunas zonas de consistencia gelatinosa; se pueden observar también zonas finamente esponjosas, moteadas algunas de ellas con degeneración hemorrágica o quística.

Su estructura microscópica es característica en grado sumo y el diagnóstico depende de la imagen histológica. Casi siempre el estroma es compacto con abundantes células fibrosas, pero en ocasiones puede ser adenomatoso y aún francamente mixomatoso. Los elementos epiteliales se aprecian como acúmulos de acinos bien definidos, si bien con diversos grados de mucificación de los elementos epiteliales. La mucina puede romper la pared del acino y pasar al estroma; este acúmulo de secreción en el estroma y en el citoplasma determina rechazo y aplanamiento de los núcleos sobre la membrana celular originando las llamadas "células de anillo de sello" de gran valor diagnóstico. A su presencia obedece el nombre de "carcinoma mucocellulare" $(8,17)$.

La ascitis se encuentra en el 50\% de los casos (9). Pueden producirse metástasis a pulmones, pleura, hígado, páncreas y huesos largos.

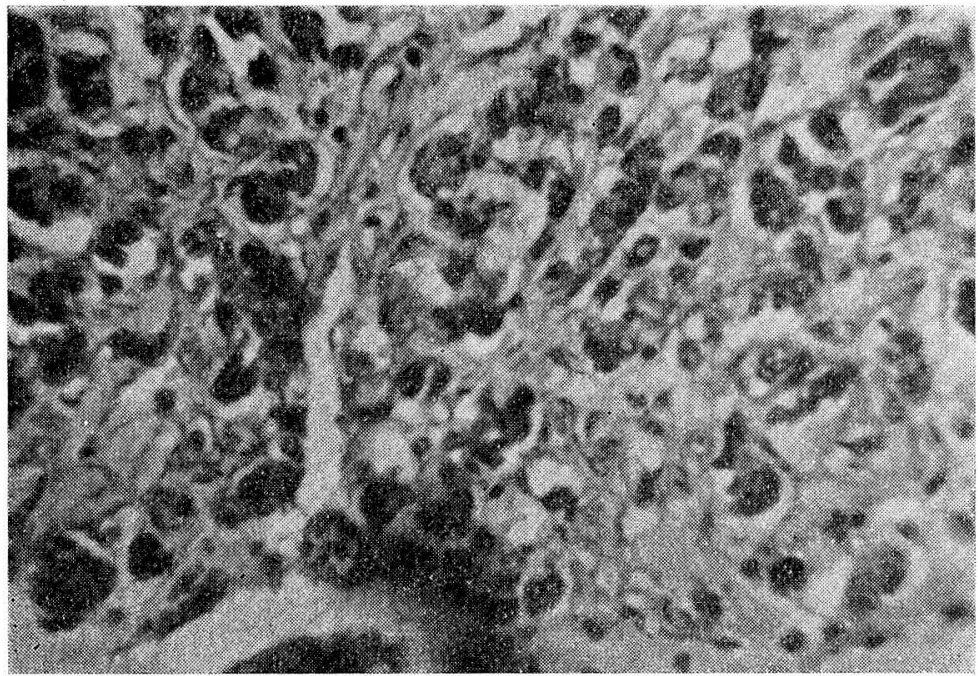

FIGURA 1 - Se aprecian las "células en anillo de sallo" características del tumor de Krukenberg. (M. 5962)

\section{Propagación}

Se aceptan cuatro vías de acceso para la propagación al ovario:

1. Implantación directa de las células tumorales sobre la superficie del ovario, células que para llegar desde su punto de origen hasta la pelvis deben usar como vehículo el exudado peritoneal; la época más propicia de implantación podría ser el día de la ovulación o de ruptura folicular.

2. Vía linfática. Los linfáticos del ovario desembocan en los ganglios lumbares y éstos se encuentran íntimamente entrelazados con los linfáticos retroperitoneales, los que se hallan invadidos en la mayoría de las pacientes por la neoplasia maligna gastrointestinal. Aun cuando Schramm 
ha observado en una misma paciente grupos de células tumorales en los linfáticos de la pared gástrica a nivel del tumor, en los linfáticos subpilóricos y en los linfáticos del ligamento tubo ovárico y del hilio del ovario (1), y aun cuando el mecanismo retrógrado de diseminación linfática ha sido demostrado en muchos puntos y acogido por los patólogos, no todos aceptan al diseminación retrógrada a lo largo de la red linfática retroperitoneal que llega al estómago procedente de los linfáticos del ovario.

3a Vía sanguínea. Es una vía que se puede admitir sin que existan pruebas histológicas significativas. Ribert sostiene que a esta vía se le puede hacer responsable de un mayor número de casos de tumores de Krukenberg que a la vía linfática.

4. Extensión directa. Sería muy difícil explicar la invasión por propagación directa a partir de un carcinoma gastrointestinal; en cambio sería muy lógico aceptarla a partir del recto y aún, si bien raras veces, del sigmoide, órganos que al adherirse a los ovarios harían posible la propagación por vecindad y contacto.

Muchas otras hipótesis se han invocado como la olcalización electiva en el ovario y hasta el citotropismo particular (1).

\section{Actividad hormonal}

Se ha discutido si este tumor tiene o no actividad hormonal. Turenen fue el primero en sugerir la posibilidad de que un tumor de Krukenberg pudiese elaborar hormonas estrogénicas y androgénicas; presentó dos pacientes post-menopáusicas con sangrado uterino por hiperplasia quística de endometrio y 17 cetosteroides altos; después de la extirpación del itumor los estrógenos llegaron a nive- les normales lo mismo que los $17 \mathrm{ce}$ tosteroides (25). Luego Scully en 1961 publicó tres nuevos casos con posible actividad estrogénica (22). No hay duda de que la reacción estrogénica que acompaña a los tumores de Krukenberg puede ser muy marcada hasta el punto de simular una tecomatosis, pero no se le puede identificar con función endocrina alguna. Spadoni afirma que hasta 1965 nueve mujeres portadoras de tumor de Krukenberg concomitante con embarazo habían dado productos con síntomas de virilización (24). Ober al estudiar una paciente encontró estroma funcionante (19).

\section{Tratamiento}

A nuestra paciente le practicamos histerectomía total con salpingo-ooforectomía bilateral; rehusó la radioterapia posterior.

La conducta difiere en diferentes instituciones. En el Instituto Nacional de Cancerología, de Bogotá, una vez descartada la participación de las vías digestivas, lo tratan como a cualquier adenocarcinoma ovárico: cirugía (histero anexectomía bilateral) en los casos operables, seguida de radioterapia a todo el abdomen y pelvis en caso de que por el tamaño del tumor y el estado de la serosa se considere justificado. En casos inoperables hacen cirugía parcial según lo logre el cirujano; irradian luego y practican más tarde una reintervención que en muchas ocasiones les permite realizar una extirpación completa (7).

Algunos autores franceses afirman que el pronóstico es tan desfavorable y la supervivencia tan efímera cuando se usa solamente la cirugía como cuando se aplica radioterapia postquirúrgica; practican sólamente histerectomía total con salpingo ooforectomía bilateral $(3,16)$. 
Hay quienes preconizan la cobaltoterapia postquirúrgica pero el Krukenberg secundario viene primordialmente del tubo gastrointestinal y por lo tanto mal se puede usar cobaltoterapia cuando ella no tiene efecto en los cánceres del aparato digestivo (3).

\section{Comentarios}

Se debe practicar una exploración pélvica sistemática durante el acto quirúrgico a toda paciente con carcinoma gastro-cisto-éntero-cólico, lo que dará la posibilidad de descubrir tumores ováricos metastásicos uni o bilaterales; si esta exploración resultare negativa, se deberán practicar posteriores y repetidos exámenes ginecológicos.

Para algunos autores es tan estrecha la relación entre carcinoma del sigmoide y metástasis ovárica que practican la histerectomía total con salpingo ooforectomía bilateral como un paso regular en el tratamiento quirúrgico de dicho carcinoma (21).

El hallazgo de un tumor de Krukenberg del ovario nos obliga también a investigar cuidadosamente la posibilidad de una neoplasia primaria en senos 0 en tracto gastrointestinal, si ella no ha sido sospechada o diagnosticada con anterioridad.

El tratamiento quirúrgico, como en todos los tumores malignos de ovario, no tiene un valor real terapéutico, pero sí puede proporcionar, entre más amplio sea, una supervivencia mayor, si bien existe la posibilidad de producir una extensión del proceso carcinomatoso.

\section{Resumen}

El tumor de Krukenberg del ovario es una entidad poco frecuente entre nosotros; en cinco hospitales del país encontramos una incidencia promedio de $6,63 \%$ en relación con los tumores malignos de ovario.

Recientes estudios sugieren una actividad hormonal del tumor de Krukenberg del ovario.

Todo cirujano que intervenga un carcinoma del aparato digestivo (gastro-cisto-éntero-cólico) debe practicar un examen de ovarios a cielo abierto con miras a descubrir oportunamente un Krukenberg secundario. Si constata la metástasis, debe intervenir de inmediato. Si la invasión ovárica no es evidente, se practicarán posteriormente exámenes ginecológicos con alguna periodicidad. Conducta similar se observará al intervenir carcinoma mamario.

El tratamiento del Krukenberg es quirúrgico: histerectomía total más salpingo ooforectomía bilateral seguido o nó de radioterapia. Está controvertido el tratamiento actínico postquirúrgico, pues mientras algunas escuelas lo preconizan como indispensable, otras lo consideran inútil.

\section{Summary}

The Krukenberg tumor of the ovary is a rare event amongst us; the average incidence found by us in five hospitals of the country gave $6.63 \%$ with regards to other malignant ovary tumors.

Recent studies sugest a hormonal activity of the Krukenberg ovary tumor.

Any surgeon operating a carcino$m a$ in the digestive apparatus (gastro-cysto-entero-colic) must perform an open examination of the ovaries in order to timely discover a secondary Krukenberg. Should metastasis be evidenced, an immediate intervention is required. Should the ovary in- 
vasion not be evident, future gynecological examinations must be performed periodically. A similar action must be taken when operating a mammary carcinoma.

The treatment of the Krukenberg is surgical; total hysterectomy plus bilateral salpingo-oophorectomy followed or not by radio-therapy. The post-surgical actinic treatment is under discussion since while certain schools consider is indispensable, others consider same useless.

\section{BIBLIOGRAFIA}

1 AMELINE, A. et SENEZE, J. Tumeurs des annexes. Dans Encyclopédie Méd. Chirurg. Gynécologie II, 680 A 10, 3, 1959.

2 ANCES, I. G. and col. Metabolism of testosterone by virilizing Krukenberg tumor of the ovary. Am. J. Obstet. Gynec. 100: 1062, 1968.

3 BOINET, R., et col. A propos d'un cas de tumeur de Krukenberg. Comptes Rendus de la Soc. Franc. de Gyn. 39: 171, 1969.

4 DIDDLE. Cáncer. 8: 1026, 1955.

5 DULCEC, J. J. Comunicación personal. Marzo $17,1971$.

6 FOX, L. P. and col. Krugenberg tumor complicating pregnancy; report with a case with androgenic activity. Am. J. Obstet. Gynec. 92: 702, 1965.

7 GAITAN, M. Comunicación personal. Agosto $13,1971$.

8 HERTIG, A. and GORE, H. Krukenberg tumor. In Tumors of the female sex organs. Part 3. Tumors of the ovary and fallopian tube. Atlas of tumors Pathology. Arm. Forces Inst. of Pathol. Washington, 1961.

9 JOSHI, V. V. Primary Krukenberg tumor of ovary. Reviem of literature and case report. Cancer. 22: 1199, 1968.

$10 \mathrm{KARSH}, \mathrm{J}$. Secondary malignant disease of the ovaries. A study of 72 autopsies. Am. J. Obstet. Gynec. 61: 1199, 1968.

11 LINARD, R. Carcinomes métastatiques bilatéraux des ovaires ou tumeurs de Krukenberg. J. de Chir. 62: 15, 1946.

12 LOPEZ, R. y CASTELLANOS, J. Comunicación personal. Marzo 2 de 1971.
13 LAWRENCE, W. D., LARSON, P. N. and HAUGE, E. T. Primary Krukenberg tumor of the ovary in pregnancy. Obstet. Gynec. 10: 54, 1957.

14 LLINAS, J. P. Tumores de Krukenberg. Rev. Médica de Colombia, Bogotá. 2: 73, 1931.

15 MULLER, P. and col. A propos of Krukenberg's tumor in a 14 years old girl. Bull. Féd. Gynéc. Obstet. Franc. 17: 537, 1965.

16 NOVAK, E. R., JONES, G. S. Y JONES, Jr. H. W. Tumor de Krukenberg. En Tratado de Ginecología. Ed. Interamericana S. A. México, Octava Ed. Pág. 491, 1971.

17 NOVAK, E. R. y WOODRUFF, J. D. Tumor de Krukenberg del ovario. En Ginecología y Obstetricia. Tratado Anatomopatológico, clínico y endocrinológico. Ed. Alhambra S. A. Madrid, pág. 372, 1964.

18 NOVAK, E. and GRAY, L. A. Krukenberg tumors of the ovary. Clinical and pathological study of 21 cases. Surg. Gynec. and Obstet. 66: 157, 1938.

19 OBER, W. B. and col. Krukenberg tumor with androgenic and progestational activity. Am. J. Obstet. Gynec. 84: 739, 1962.

20 OCAMPO, L. B. Comunicación personal. Marzo 2 de 1971.

21 PARSONS, L. and SOMERS, S. C. Metastatic (secondary) ovarian neoplasms. In Gynecology. Ed. W. B. Saunders, Co. Philadelphia, pág. 811, 1964.

22 SCULLC, R. E. and RICHARDSON, G. S. Cancer. 14: 827, 1961.

23 SOLOWAY, J. and col. Krukenberg tumors of the ovary. Obstet. Gynec. 8: 363, 1956.

24 SPADINI. L. R. and col. Virilization coexisting with Krukenberg tumir during pregnancy. Am. J. Obstet. Gynec. 92: 981, 1965.

25 TURENEN, K. Hormonal secretion of Krukenberg tumors. Acta Endocrinol. 20: 50, 1955.

26 VALENZUELA, S., ZARATE, A., DIAZ, A., DELGADO, J. y MCGREGOR, C. Revisión de 492 casos de neoplasias ováricas estudiadas en un período de once años. Ginec. Obstet. Méx. 196: 125, 1973.

27 WOODRUFF, J. D. and NOVAK, E. R. Krukenberg tumors of the ovary. Obstet. Gynec. 15: 351, 1960. 